Supporting Information

\title{
Helical Magnetic Field-Induced Real-Time Plasmonic Chirality Modulation
}

Ki-Jae Jeong ${ }^{1}$, Dong Kyu Lee ${ }^{1}$, Van Tan Tran², Caifeng Wang ${ }^{1}$, Jiawei Lv³, Jinhae Park Zhiyong Tang ${ }^{3}$, Jaebeom Lee ${ }^{5 *}$

${ }^{1}$ Department of Cogno-Mechatronics Engineering, Pusan National University, Busan 46279, Republic of Korea.

${ }^{2}$ Research Institute of Materials Chemistry, Chungnam National University, Daejeon, 34134, Republic of Korea.

${ }^{3}$ CAS Key Laboratory for Nanosystem and Hierarchy Fabrication, CAS Center for Excellence in Nanoscience, National Center for Nanoscience and Technology, Beijing, 100190, China

${ }^{4}$ Department of Mathematics, Chungnam National University, Daejeon, 34134, Republic of Korea.

${ }^{5}$ Department of Chemistry, Chungnam National University, Daejeon, 34134, Republic of Korea.

E-mail: nanoleelab@cnu.ac.kr 


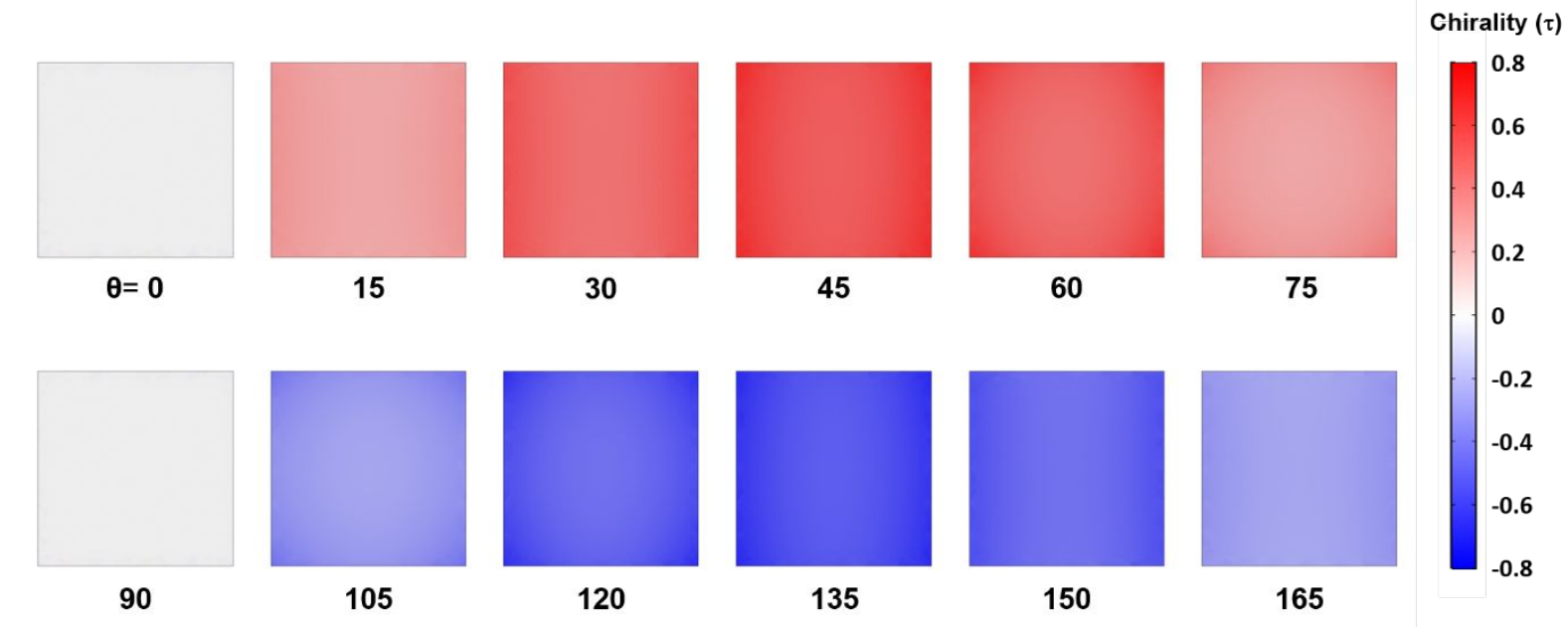

Figure S1. Calculated chirality of $h \mathrm{~B}$ along the z-axis and projected to the [001] plane depending on $\theta$ based on $\tau=\int n \cdot \nabla \times n$, where LH and RH are colored as red and blue, respectively. Note that at the $\theta$ of $0^{\circ}$ and $90^{\circ}$, it is achiral since the parallel magnetic field is generated while the others are chiral depending on the range of angle i.e., LH: $0^{\circ}<\theta<90^{\circ}$, while RH: $90^{\circ}<\theta<180^{\circ}$. 


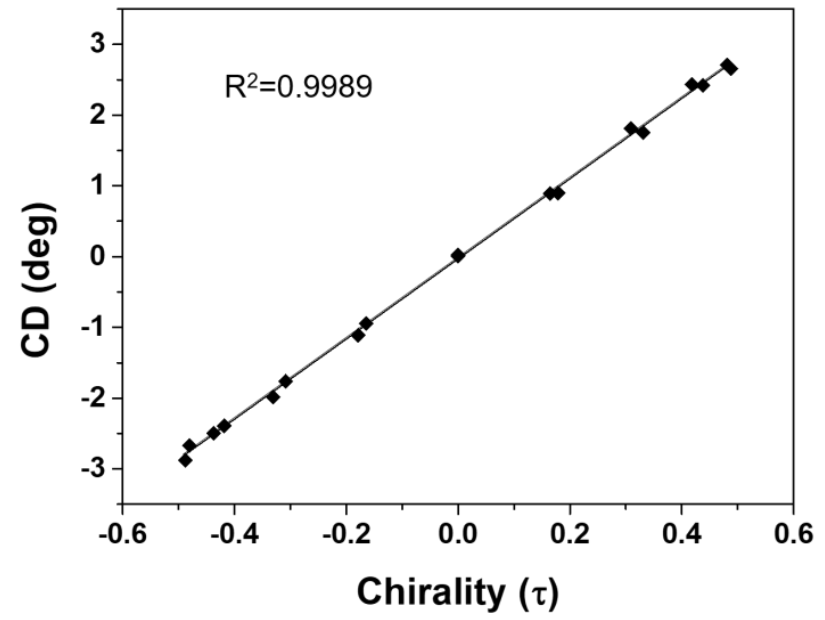

Figure S2. Linearity plot between CD intensity at $770 \mathrm{~nm}$ and chirality of $h \mathrm{~B}$.
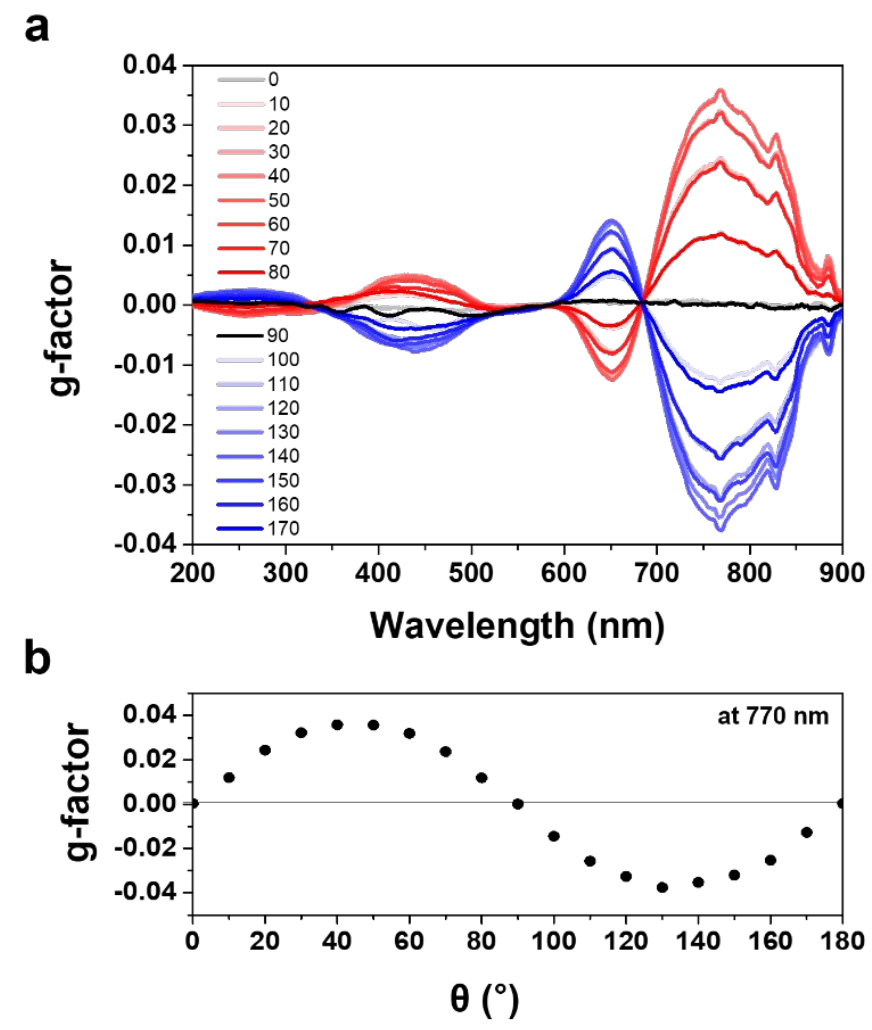

Figure S3. The g-factor spectrum of MagPlas-I depending on the rotation angle of magnets.

The chiroptical anisotropy g-factor was calculated using the equation;

$$
g-\text { factor }=\frac{2\left(A_{L C P}-A_{R C P}\right)}{\left(A_{L C P}+A_{R C P}\right)}
$$




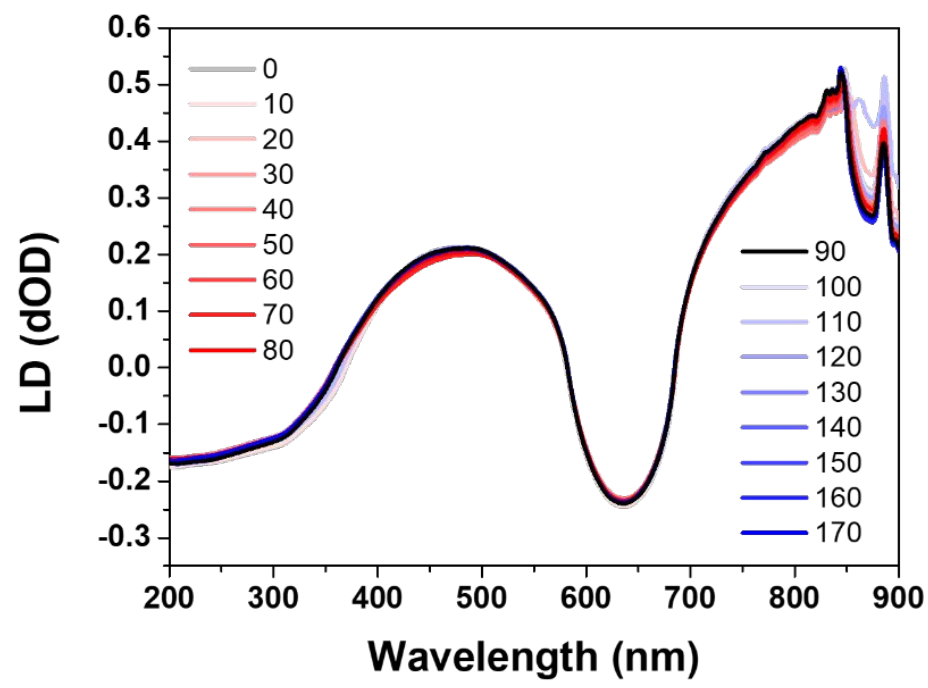

Figure S4. Linear dichroism (LD) spectrum of MagPlas-I depending on the rotation angle of magnets.
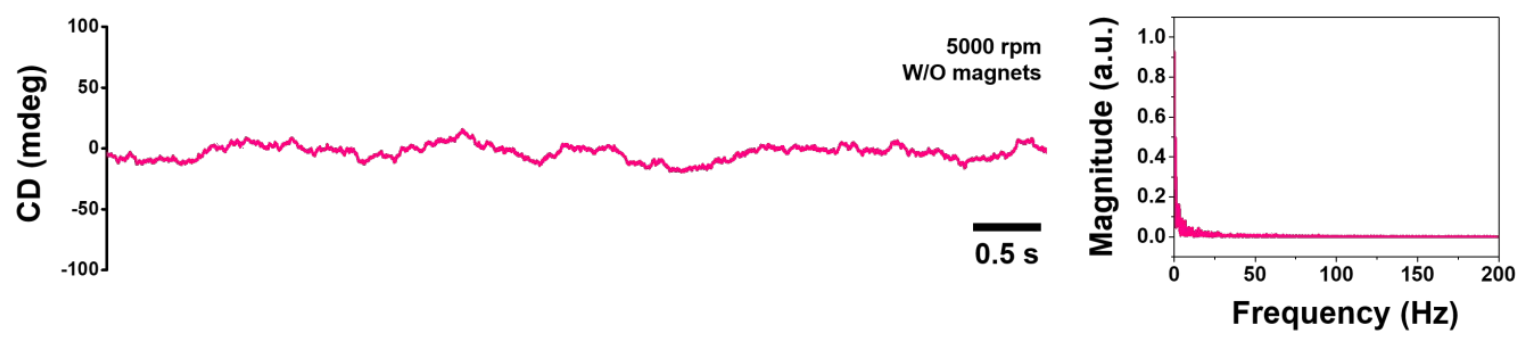

Figure S5. Time-dependent CD spectrum under rotation of motors with $5000 \mathrm{rpm}$ without magnets. 
a

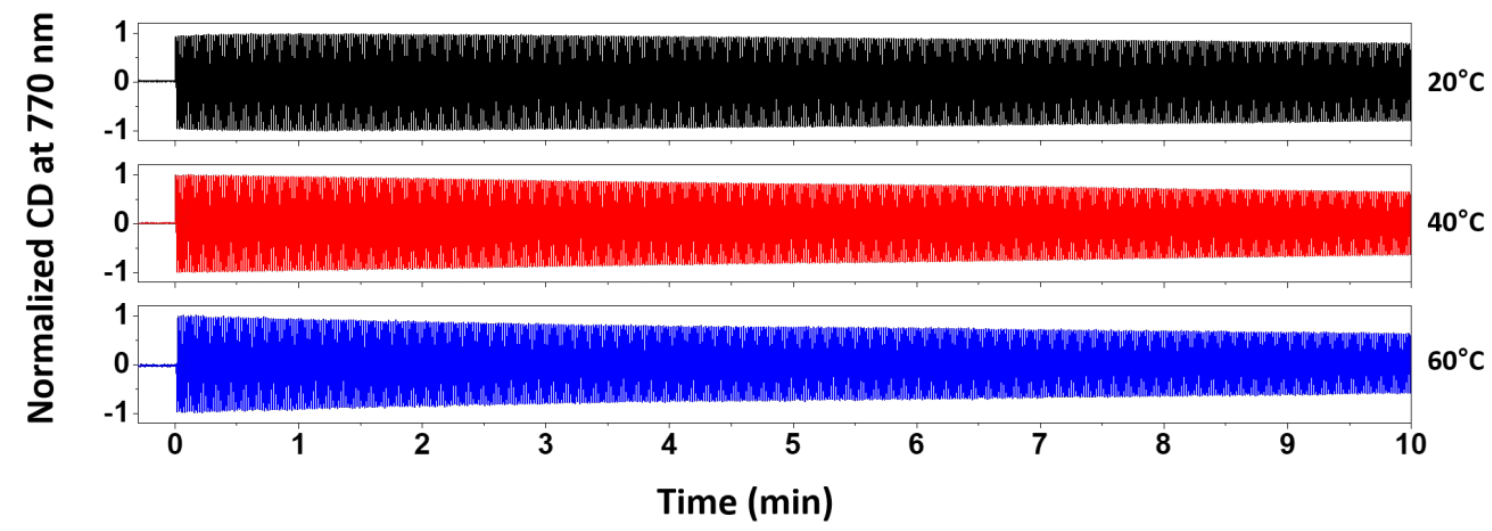

b
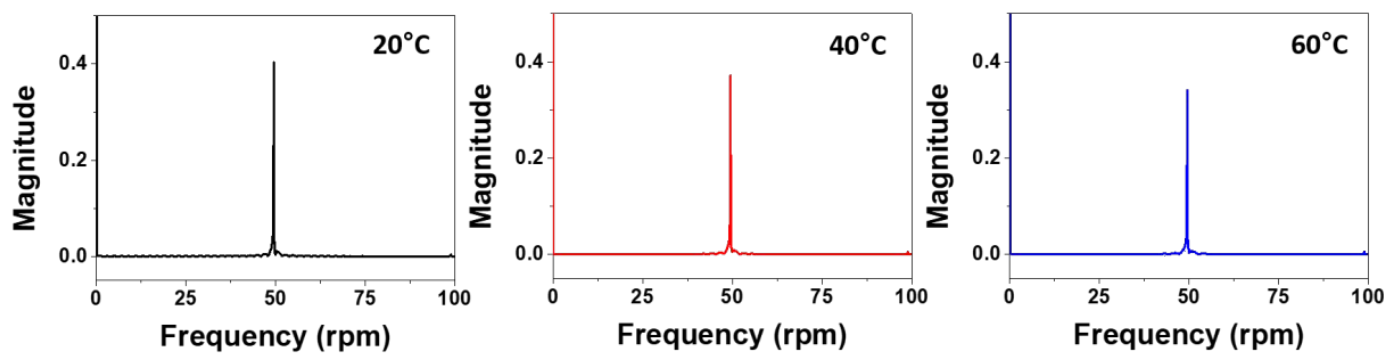

Figure S6. Chirality tuning ability at different temperature conditions. (a) Time-based CD signal monitoring at three different temperature conditions $\left(20,40\right.$, and $\left.60^{\circ} \mathrm{C}\right)$ demonstrating chirality modulation conservation over various temperatures. (b) Fourier transformed graphs of each temperature condition showing a constant frequency of $49.45 \mathrm{rpm}$. 

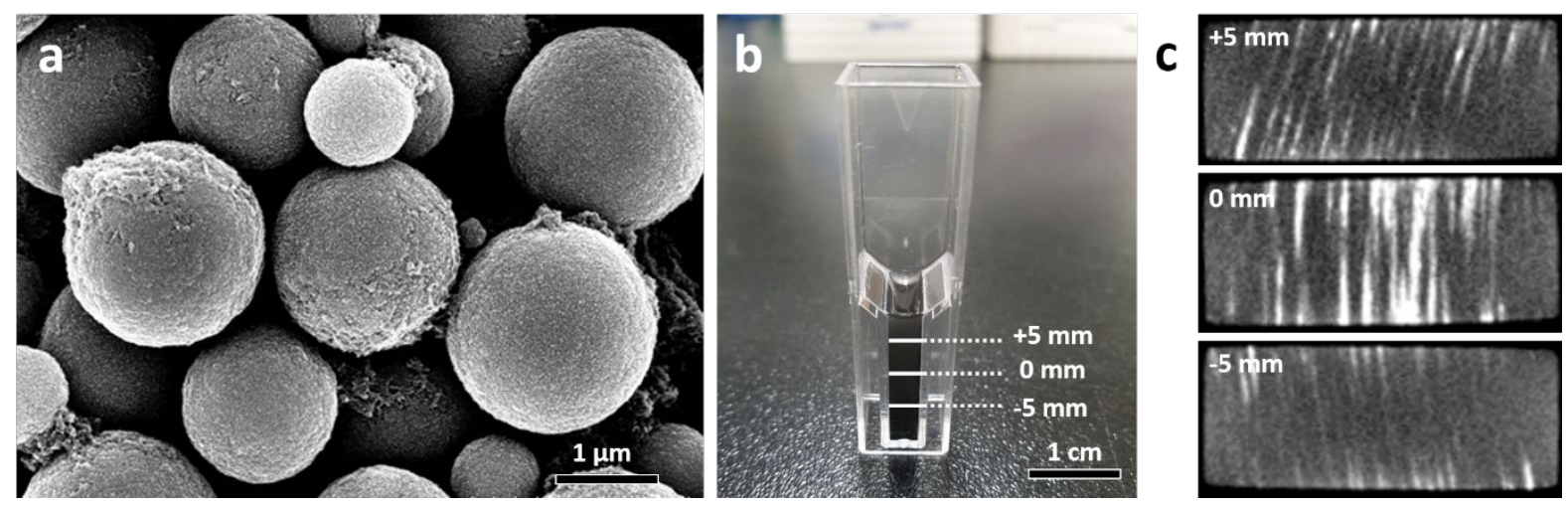

Figure S7. Structure analysis of assembled magnetic nanochain under $h \mathbf{B}$. (a) SEM image of micrometer-sized $\mathrm{Fe}_{3} \mathrm{O}_{4}$ magnetic nanoparticles (MNPs) for single-photon emission computed tomography (SPECT) imaging. (b) Photograph of cured PDMS containing micro-sized MNPs in a cuvette. (c) 2D SPECT images of PDMS (LH structures) at three different z-positions. The bright lines in the SPECT images show aligned magnetic nanochains. The aligned chain direction is changed depending on z-position, presenting LH helical structures. 
a
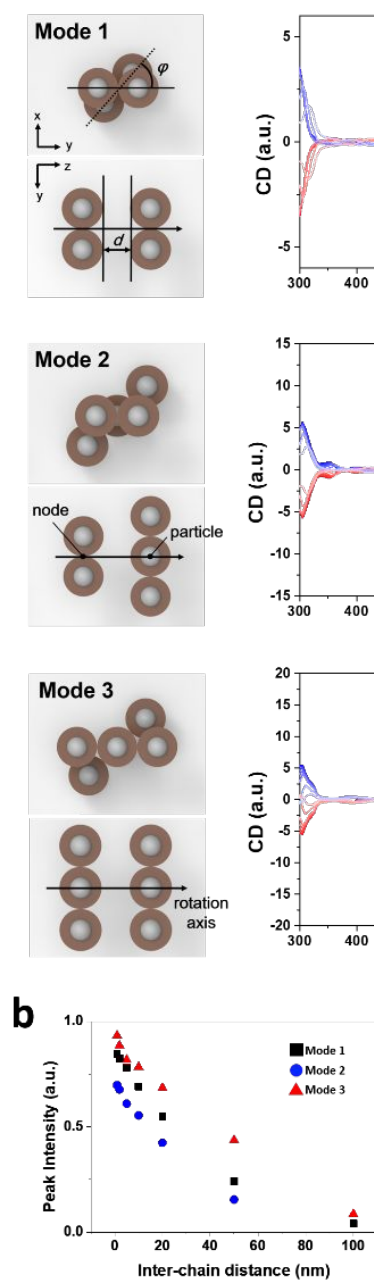

$15^{\circ}$
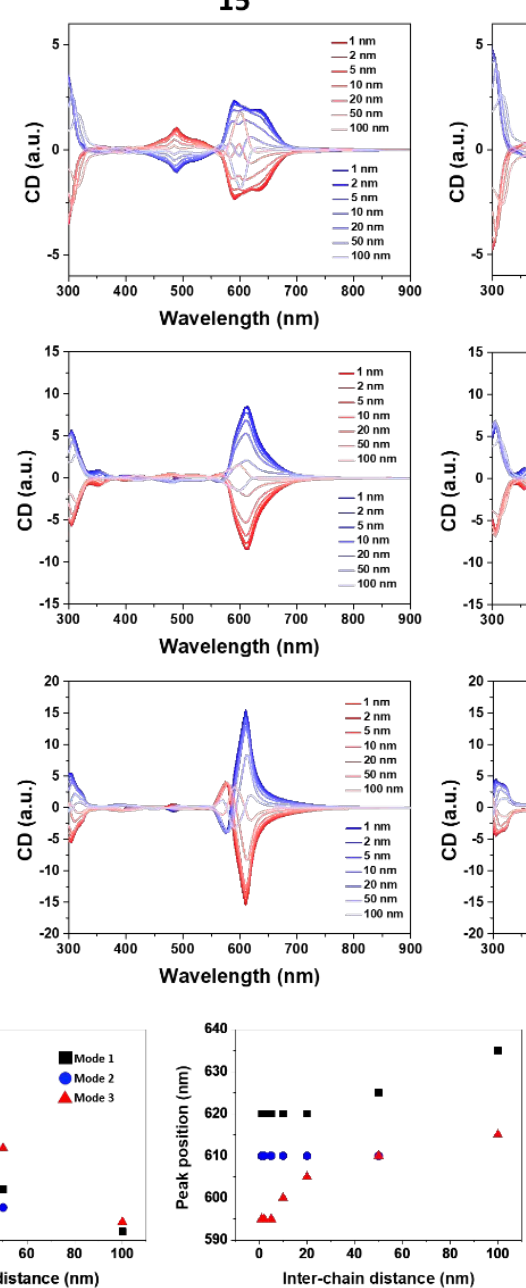
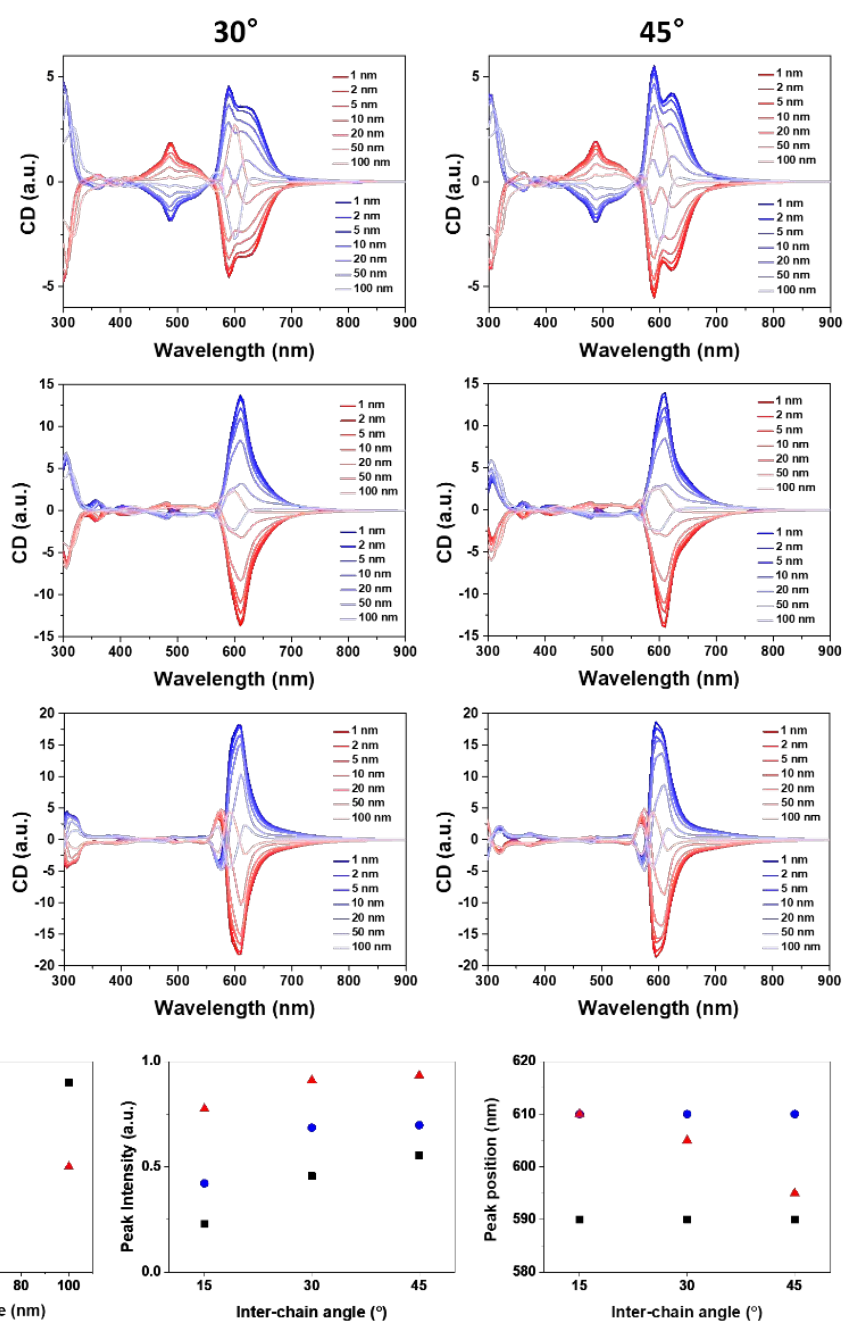

Figure S8. Optical simulation results of (a) CD spectra depending on the arrangement mode, inter-chain angle $(\varphi)$, and inter-chain distance $(d)$. (b) The 2-D plot chart of the peak intensity and position variation with $d$ and $\varphi$. The optical simulation was conducted by varying $\varphi$ and $d$ depending on three representative skew line configurations (Mode 1: node-node, Mode 2: node-particle, Mode 3: particle-particle). The spectral intensity increases as $\varphi$ increased up to $45^{\circ}$, which is consistent with experimental results. When $d$ is less than $50 \mathrm{~nm}$, the difference in CD spectrum shapes between modes is clear. However, at $100 \mathrm{~nm}$, the difference between Modes disappeared. Spectral results in all modes are analogous to experimental results, indicating that the $d$ of helical nanochain structures is greater than $100 \mathrm{~nm}$. When $d$ is increased, the peak intensity is decreased and exhibits a red-shift. 

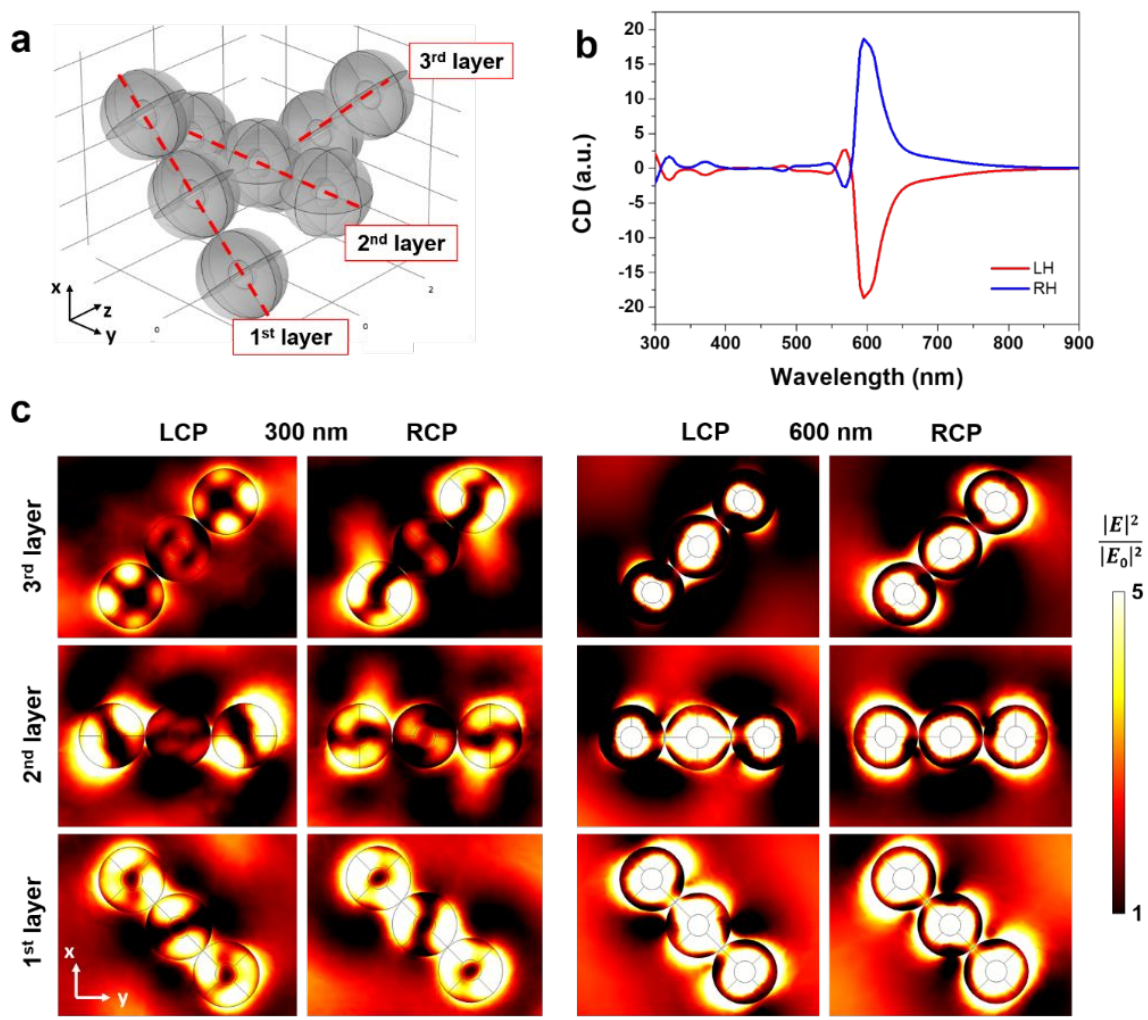

Figure S9. Light-matter interaction of MagPlas nanochains. (a) The structural arrangement of MagPlas nanochains (LH). (b) The CD spectrum results based on theoretical calculation. The computational simulation was carried out in the condition of the vacuum state observe the maximum influence of the electric field into the nanochain arrangement under the propagation of LCP and RCP light. (c) Electric field distribution in structures with each LCP and RCP at 300 and $600 \mathrm{~nm}$. Each layer contains a red dashed line in (a). In this helical chain geometry, electric field enhancement by propagating LCP and RCP is noticeably different at $600 \mathrm{~nm}$. At $600 \mathrm{~nm}$, where the highest CD peak was observed, the electric field enhancement at the third layer is uniquely stronger by RCP than by LCP, suggesting that plasmonic coupling is generated differently by LCP and RCP and induces unique chiroptical properties. The strong $\mathrm{CD}$ intensity at $600 \mathrm{~nm}$ originates from asymmetric plasmonic resonance, and CD intensity at $300 \mathrm{~nm}$ is the asymmetric interaction with iron oxide shell. 

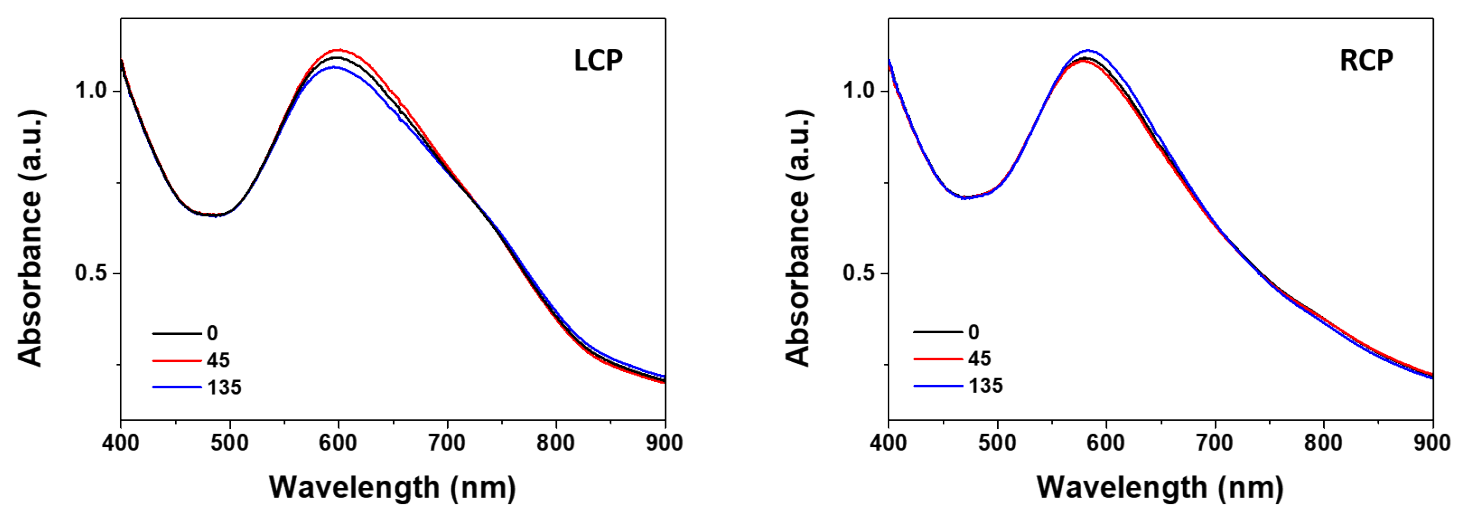

Figure S10. The LCP and RCP absorbance spectrum of assembled structures (MagPlas-II). When LCP is propagated, it interacts differently with LH- and $\mathrm{RH}$-structures due to asymmetric plasmonic coupling. In the absorbance spectrum of LCP, the plasmonic resonance peak intensity is highest in LH structures and lowest in $\mathrm{RH}$ structures. When RCP propagates through the structures, those interactions are reversed, exhibiting the highest absorption in $\mathrm{RH}$ structures and lowest in LH structures. 


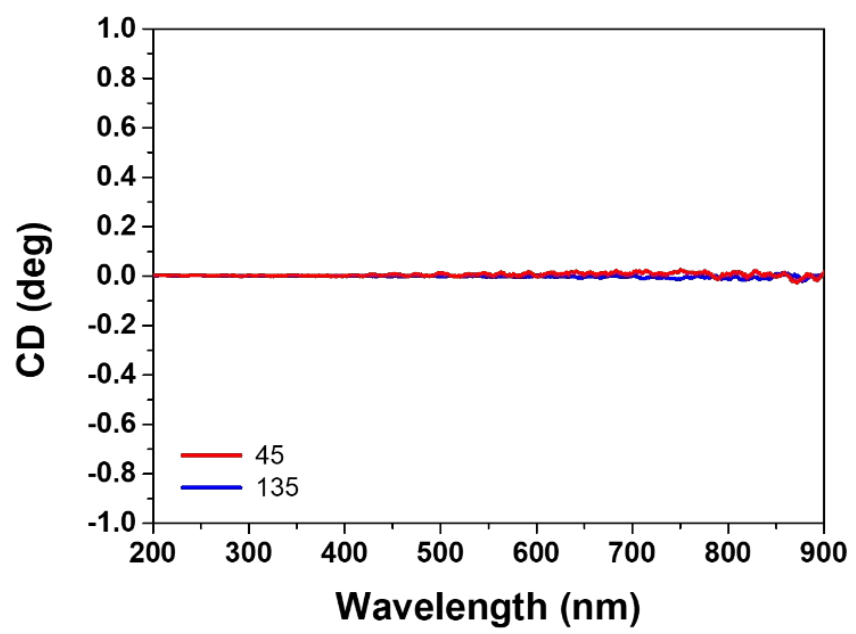

Figure S11. CD spectrum of $200 \mathrm{~nm}$-sized magnetic nanoparticles (MNPs) under $h \mathrm{~B}\left(\theta=45^{\circ}\right.$ and $\left.\theta=135^{\circ}\right)$.

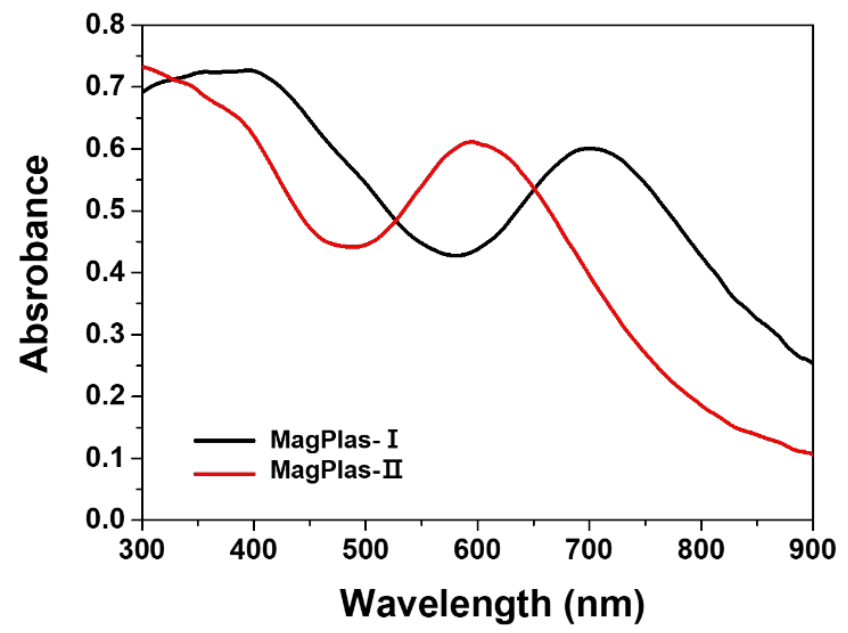

Figure S12. UV-Vis spectra of MagPlas-I and MagPlas-II. 
a

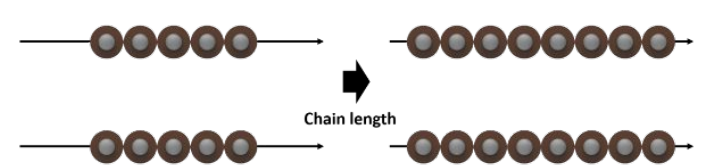

b
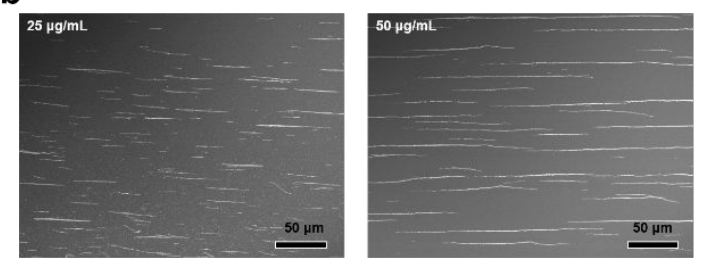

C

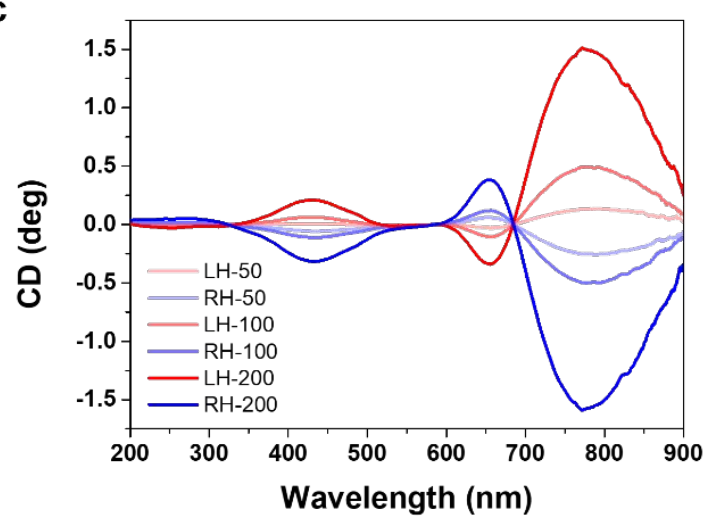

Figure S13. Experimental data of MagPlas NPs concentration-dependent CD spectra. (a) Illustration of the concentration effect on MagPlas nanochain assembly. (b) SEM images of MagPlas nanochains assembled with different concentration of NPs solution (25 and 50 $\mu \mathrm{g} / \mathrm{mL}$ ). (c) Concentration-dependent CD spectra, where the numbers in the legend depict the concentration of the MagPlas NPs solution $(\mathrm{mg} / \mathrm{mL})$. 